\title{
Guest-Editorial
}

\section{Scientific Workflows}

\author{
Adam Belloum $^{\mathrm{a}}$, Ewa Deelman ${ }^{\mathrm{b}}$ and Zhiming Zhao ${ }^{\mathrm{a}}$ \\ ${ }^{a}$ University of Amsterdam, Amsterdam, The Netherlands \\ ${ }^{\mathrm{b}}$ USC Information Sciences Institute, Marina del Rey, CA, USA
}

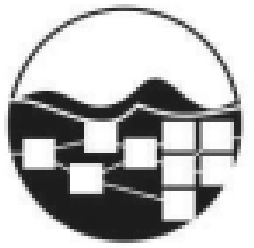

In recent years workflows have emerged as a key technology that enables large-scale computations on distributed resources. Workflows enable scientists to design complex applications that are composed of individual application components or services. Often times these components and services are designed, developed, and tested collaboratively. Because of the size of the data and the complexity of the analysis, large amounts of shared resources such as clusters and storage systems are being used to store the data sets and execute the workflows. The process of workflow design and execution in a distributed environment can be very complex and involve mapping high-level workflow descriptions onto the available resources, as well as monitoring and debugging of the subsequent execution. Because computations and data access operations are performed on shared resources, there is an increased interest in managing the fair allocation and management of those resources at the workflow level.

Adequate workflow descriptions are needed to sup- port the complex workflow management process that includes workflow creation, reuse, and modifications made to the workflow over time - for example modifications to the individual components. Additional annotations may provide guidelines and requirements for resource mapping and execution.

Large-scale scientific applications impose requirements on the workflow systems. Besides the magnitude of data processed by the workflow components, the resulting and intermediate data need to be annotated with provenance information and any other information needed to evaluate the quality of the data and support the repeatability of the analysis.

This special issue of the Scientific Programming Journal is dedicated to Scientific Workflows and includes research that was presented at two workshops devoted to topic: The International Workshop on Workflow Systems in e-Science (WSES 06) and The Workshop on Workflows in Support of Large-Scale Science (WORKS 06).

The papers address various aspects of scientific workflows including issues of workflow scheduling, quality of service, service composition, and the application of workflows in science. We hope that this issue is informative and of interest to workflow researchers as well as application scientists. 

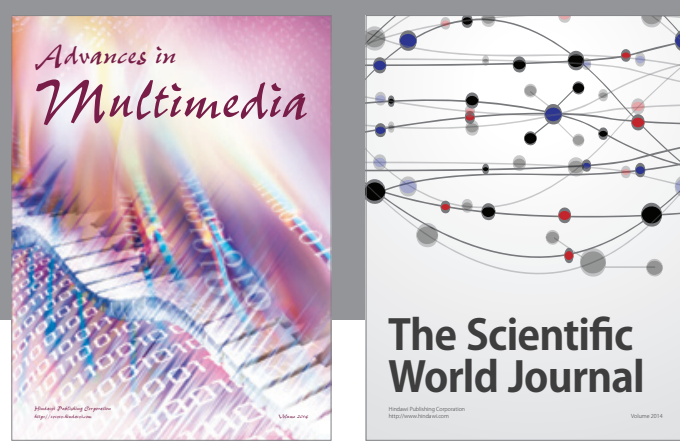

The Scientific World Journal
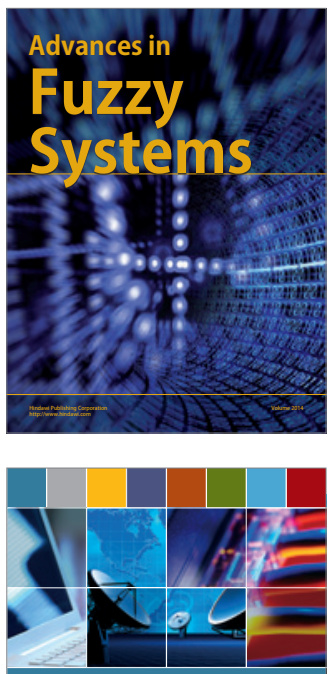

Computer Networks and Communications
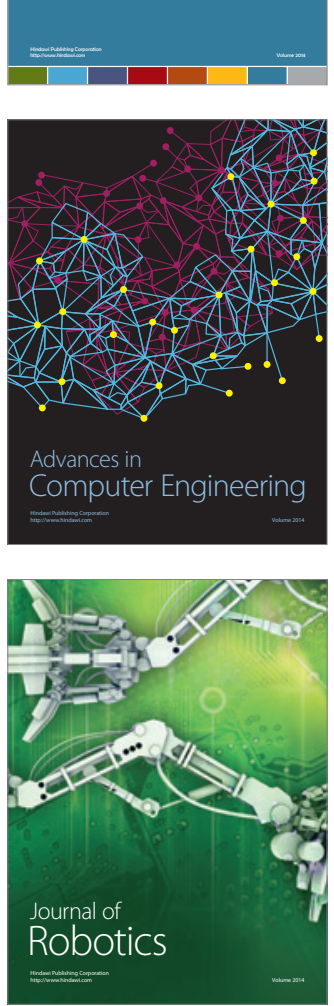
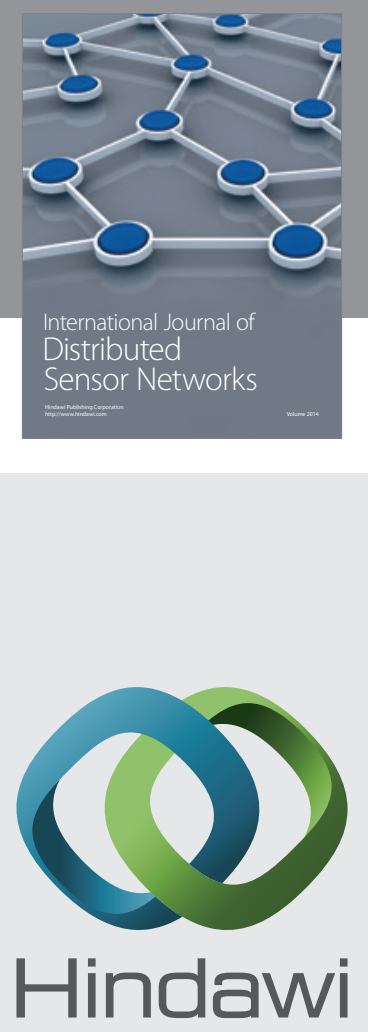

Submit your manuscripts at

http://www.hindawi.com
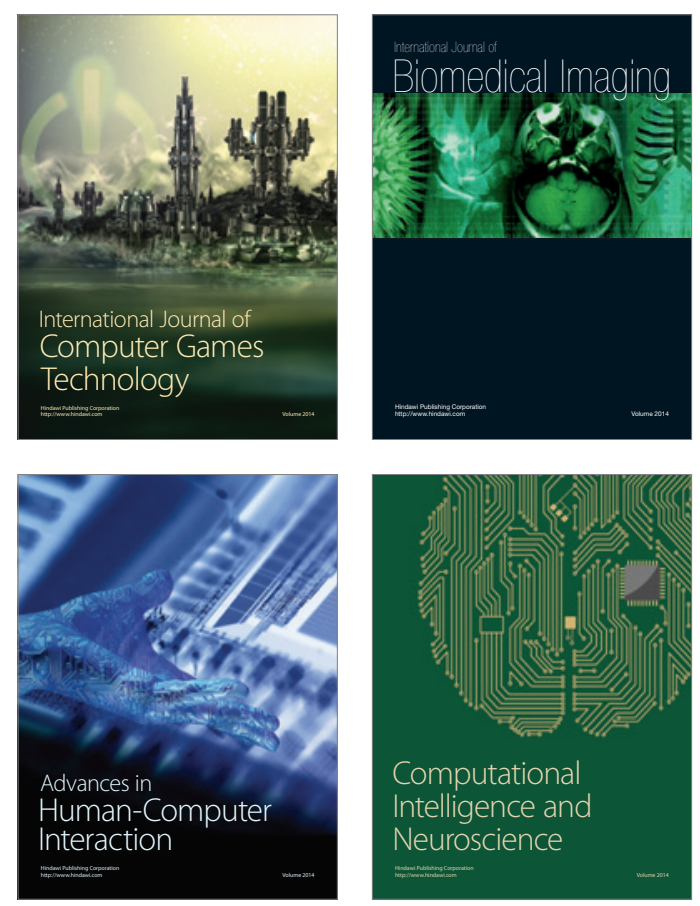
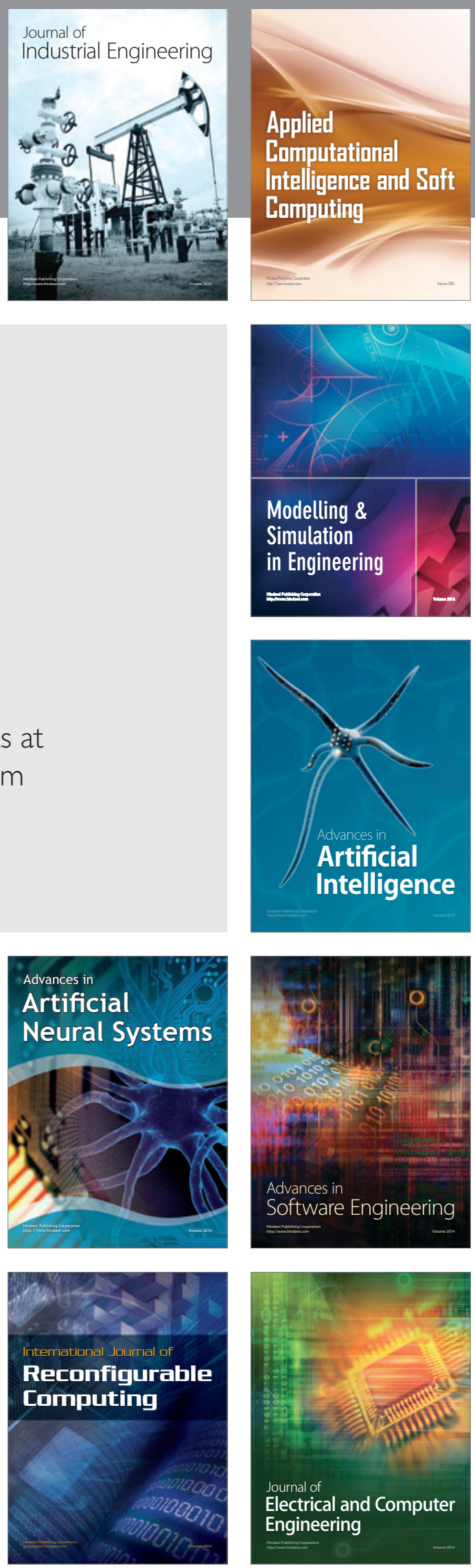